\title{
Development of an Online Home Appliance Control System Using Augmented Reality and an SSVEP- Based Brain-Computer Interface
}

\author{
Seonghun Park, Ho-Seung Cha, Jinuk Kwon, Hodam Kim and Chang-Hwan Im \\ Department of Biomedical Engineering \\ Hanyang University \\ Seoul, Republic of Korea \\ ich@,hanyang.ac.kr
}

\begin{abstract}
In this study, we implemented a new home appliance control system by combining a steady-state visual evoked potential (SSVEP)-based brain-computer interface (BCI), augmented reality (AR), and internet of things (IoT) technologies. The visual stimuli were presented on a see-through headmounted display (HMD), while the recorded brain activity was analyzed to classify the control command, and the home appliances were controlled through IoT. The average classification accuracy of the SSVEP-BCI-based control system was $92.8 \%$, with an information transfer rate (ITR) of 37.4 bits/min. The proposed system exhibited an excellent performance, surpassing the best results reported in previous studies regarding external device control based on BCI using an HMD as rendering device.
\end{abstract}

Keywords-augmented reality, brain-computer interface, electroencephalography, internet of things, steady-state visual evoked potential

\section{INTRODUCTION}

Brain-computer interfaces (BCIs) have been emerging as a promising tool to assist daily life activities of the elderly and the people with physical disability for past decades. Recently, with the outstanding advances in the augmented reality (AR) device and internet of things (IoT) technology, a home appliance control system often referred to as "smart home" has become an attractive option to aid the daily tasks of those people. In this study, we implemented a new home appliance control system by combining electroencephalography (EEG)based BCI, AR, and IoT.

\section{METHODS}

We adopted a steady-state visual evoked potential (SSVEP)-based BCI paradigm for the implementation of a fast and robust BCI system. In the first experiment, we compared the $\mathrm{BCI}$ performance of different types of visual stimuli in $\mathrm{AR}$ environment to determine the optimal visual stimulus. In the second experiment, with the optimal stimulus chosen in the preceding experiment, we evaluated the feasibility of the proposed system by controlling home appliances in real-time with 17 participants. The visual stimuli were presented in AR environment with a see-through head-mount display (HMD), while the recorded brain activity was analyzed to classify the

This work was supported by Institute for Information \& communications Technology Promotion(IITP) grant funded by the Korea government(MSIT) (2017-0-00432 , Development of non-invasive integrated BCI SW platform to control home appliances and external devices by user's thought via AR/VR interface).

978-1-7281-4707-9/20/\$31.00 C2020 IEEE

Authorized licensed use limited to: Hanyang University. Downloaded on February 08,2021 at 07:12:48 UTC from IEEE Xplore. Restrictions apply. control command and the home appliances were controlled through IoT (Fig. 1). For the classification of SSVEP responses, a recently developed algorithm called the extension of multivariate synchronization index [1] was adopted. Repetitive eye blinks in a short time period, identified by an electrooculogram (EOG), were used as a switch to turn the control system on/off, such that our system was operated solely by the user without aid from others.

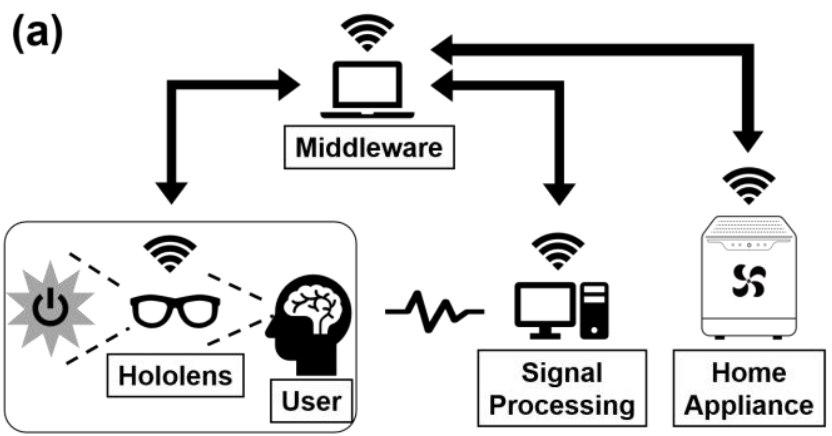

(b)

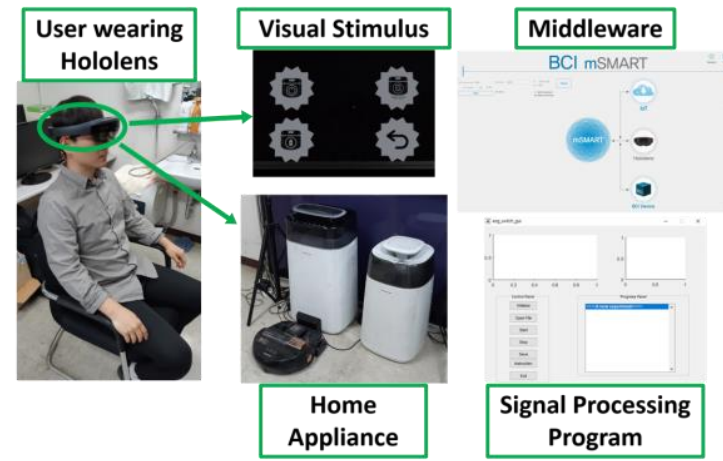

Figure 1. (a) A schematic diagram of the proposed home appliance control system. The visual stimuli were presented to the user via a Hololens, which was controlled by the middleware. The physiological signals recorded from the user were analyzed by real-time signal processing software, and then sent to the middleware. Based on the analysis results, the middleware generated an appropriate command and delivered this to the selected home appliance. The experiment was conducted in an environment in which the Hololens, middleware, signal processing program, and home appliances were all connected to the same Wi-Fi network. (b) Real pictures of the developed platform. 


\section{RESULTS}

In the first experiment, grow/shrink stimulus, a star-shaped flickering object with changing size, was selected as the optimal stimulus eliciting SSVEP response most effectively compared to the others. In the second experiment, all users could turn on/off the system whenever they want with the eyeblinking-based switch, and carried out all the device control tasks without having difficulty. It took about $2.6 \mathrm{~s}$ on average to switch on/off the system and the mean false positive rate of the switch operation was 0.015 times $/ \mathrm{min}$. Average classification accuracy of the SSVEP-BCI-based control system was $92.8 \%$ with an information transfer rate (ITR) of $37.4 \mathrm{bits} / \mathrm{min}$ (Table I). The proposed system showed excellent performance which surpassed the best results reported in the previous studies on external device control system based on BCI (Table II).

THE EXPERIMENTAL RESULTS OF EXPERIMENT II

\begin{tabular}{|c|c|c|c|c|c|}
\hline Subject & $\begin{array}{l}\text { Total } \\
\text { Trials }\end{array}$ & $\begin{array}{l}\text { Correct } \\
\text { Trials }\end{array}$ & $\begin{array}{c}\text { Incorrect } \\
\text { Trials }\end{array}$ & $\begin{array}{c}\text { Accuracy } \\
(\%)\end{array}$ & $\begin{array}{c}\text { ITR } \\
\text { (bits/min) }\end{array}$ \\
\hline P1 & 39 & 39 & 0 & 100 & 48 \\
\hline $\mathrm{P} 2$ & 44 & 40 & 4 & 90.9 & 34.0 \\
\hline P3 & 44 & 40 & 4 & 90.9 & 34.0 \\
\hline P4 & 45 & 42 & 3 & 93.3 & 37.0 \\
\hline P5 & 39 & 39 & 0 & 100 & 48 \\
\hline P6 & 39 & 39 & 0 & 100 & 48 \\
\hline P7 & 50 & 43 & 7 & 86.0 & 28.7 \\
\hline P8 & 48 & 41 & 7 & 85.4 & 28.1 \\
\hline P9 & 44 & 41 & 3 & 93.2 & 36.8 \\
\hline P10 & 40 & 39 & 1 & 97.5 & 43.0 \\
\hline P11 & 43 & 41 & 2 & 95.4 & 39.7 \\
\hline P12 & 48 & 42 & 6 & 87.5 & 30.2 \\
\hline P13 & 39 & 39 & 0 & 100 & 48 \\
\hline P14 & 41 & 40 & 1 & 97.6 & 43.1 \\
\hline P15 & 49 & 41 & 8 & 83.7 & 26.4 \\
\hline P16 & 45 & 42 & 3 & 93.3 & 37.0 \\
\hline P17 & 55 & 46 & 9 & 83.6 & 26.3 \\
\hline Mean & & & & 92.8 & 37.4 \\
\hline
\end{tabular}

\section{DISCUSSION}

With the outstanding advances in medicine, healthcare, and therapeutics, life expectancy is gradually increasing, and thus the social burden of caring for elderly and paralyzed people will soon become serious. The smart home system could provide a plausible solution to circumvent this problem. To the best of our knowledge, this is the first study which combined $\mathrm{AR}$, IoT, and BCI to develop an external device control system. Additionally, the experimental results demonstrated that the proposed BCI-based home appliance control system can be utilized in practical scenarios. In conclusion, we developed a practical self-paced home appliance control system for the people with physical disability and the elderly, which has a potential to significantly elevate their quality of life.

TABLE II

Comparison of Performances With Previous BCI-Based EXTERNAL DeVice CONTROL StUdies

\begin{tabular}{ccccc}
\hline \hline Study & $\begin{array}{c}\text { Accuracy } \\
(\%)\end{array}$ & $\begin{array}{c}\text { ITR } \\
\text { (bits/min) }\end{array}$ & $\begin{array}{c}\text { Necessity } \\
\text { of } \\
\text { Calibration }\end{array}$ & $\begin{array}{c}\text { Rendering } \\
\text { Device }\end{array}$ \\
\hline $\begin{array}{c}\text { Kishore et al. } \\
{[2]}\end{array}$ & 87 & 24.7 & Y & VR-HMD \\
$\begin{array}{c}\text { Takano et al. } \\
{[3]}\end{array}$ & 79.4 & 5.4 & Y & AR-HMD \\
$\begin{array}{c}\text { Wang et al. } \\
{[4]}\end{array}$ & 83.3 & 4.6 & Y & AR-HMD \\
$\begin{array}{c}\text { Si-Mohammed } \\
\text { et al. [5] }\end{array}$ & 90 & 8.7 & & AR-HMD \\
Proposed \\
System
\end{tabular}

\section{REFERENCES}

[1] Y. Zhang, D. Guo, D. Yao, and P. Xu, "The extension of multivariate synchronization index method for ssvep-based bci," Neurocomputing, vol. 269, pp. 226-231, Jun. 2017.

[2] S. Kishore, et al., "Comparison of ssvep bci and eye tracking for controlling a humanoid robot in a social environment," PresenceTeleoper. Virtual Env., vol. 23, no. 3, pp. 242-252, Oct. 2014.

[3] K. Takano, N. Hata, and K. Kansaku, "Towards intelligent environments: An augmented reality-brain-machine interface operated with a see-through head-mount display," Front. Neurosci., vol. 5, p. 60, Apr. 2011.

[4] M. Wang, R. Li, R. Zhang, G. Li, and D. Zhang, "A wearable ssvepbased bci system for quadcopter control using head-mounted device," IEEE Access, vol. 6, pp. 26789-26798, Apr. 2018.

[5] H. Si-Mohammed, et al., "Towards bci-based interfaces for augmented reality: Feasibility, design and evaluation," IEEE Trans. Vis. Comput. Graph. 2019. 